\section{Quantification of ${ }^{18}$ F-DCFPyL Uptake: TBR Versus Patlak's Analysis}

TO THE EDITOR: In prostate-cancer patients investigated with ${ }^{18} \mathrm{~F}$-DCFPyL, a second-generation ${ }^{18} \mathrm{~F}$-labeled prostate-specific membrane antigen ligand, Jansen et al. have recently validated the image-based tumor-to-blood ratio (TBR) as an optimal trade-off between a reliable surrogate for the net influx rate of the tracer versus simplicity of its assessment (1). The image-based TBR (blood-mL.tumor- $\mathrm{mL}^{-1}$ ) can be obtained from any standard whole-body acquisition as the mean tumor-activity concentration $\left(\mathrm{C}_{\mathrm{T}}(\mathrm{t}) ; \mathrm{kBq} \cdot \mathrm{mL}^{-1}\right)$ to the time-matched blood-activity concentration $\left(\mathrm{C}_{\mathrm{p}}(\mathrm{t}) ; \mathrm{kBq} \cdot \mathrm{mL}^{-1}\right)$ within the ascending aorta. A high correlation coefficient was found between TBR and net-influx-rate constant $(\mathrm{Ki}$; $\mathrm{mL} . \mathrm{min}^{-1} \cdot \mathrm{mL}^{-1}$ ) obtained from a reversible 2-tissue-compartment model, whereas the SUV, normalized either to body weight or leanbody mass, showed a poor SUV-Ki correlation $\left(R^{2}=0.96\right.$ vs. 0.47 and 0.60, respectively; Figure 3 and Table 3 in Jansen et al. (1)).

We believe that the comparison between either whole-blood or image-based TBR proposed by Jansen et al. and Patlak's graphical analysis may be fruitful because the former is actually the $y$-axis of the latter. For $\mathrm{t}>\mathrm{t}^{*}$ and irreversible trapping, Patlak's basic equation is indeed: $\operatorname{TBR}(\mathrm{t})=\mathrm{C}_{\mathrm{T}}(\mathrm{t}) / \mathrm{C}_{\mathrm{p}}(\mathrm{t})=\mathrm{Ki} \times \mathrm{AUC}(\mathrm{t}) / \mathrm{C}_{\mathrm{p}}(\mathrm{t})+$ $V_{b}(2)$. AUC $(t)$ is the time integral of $C_{p}(t)$ and $V_{b}$ the fraction of free ${ }^{18} \mathrm{~F}$-DCFPyL in blood and interstitial volume $\left(\mathrm{mL} \cdot \mathrm{mL}^{-1}\right)$. The ratio $\operatorname{AUC}(\mathrm{t}) / \mathrm{C}_{\mathrm{p}}(\mathrm{t})$ is the so-called stretched time $\left(\mathrm{t}_{\mathrm{s}} ; \min \right)$, and then writing $\operatorname{TBR}(\mathrm{t})=\mathrm{Ki} \times \mathrm{t}_{\mathrm{s}}+\mathrm{V}_{\mathrm{b}}$ leads to further comment on Figure 3B by Jansen et al. that shows TBR versus $\mathrm{Ki}(1)$. The linear slope of Figure $3 \mathrm{~B}\left(R^{2}=0.96\right)$ is actually an average value of $t_{\mathrm{s}}$ that is specific to Jansen et al.'s study. To support this assertion, blood data can be extracted from Figure 1 (using the WebPlotDigitizer software) to calculate AUC from trapezoidal integration and, hence, to calculate $t_{s}$. Because image-based TBR was assessed by Jansen et al. at 105-110 min after injection, when $\mathrm{t}$ is $107.5 \mathrm{~min}, \mathrm{t}_{\mathrm{s}}$ is then estimated to be $204 \mathrm{~min}$, which is consistent with the 222-min slope for the TBR-Ki correlation reported in Table 3 (1). Thus, such a crucial role of $\mathrm{t}_{\mathrm{s}}$ in the TBR-Ki correlation and, hence, that of real time t, stresses Jansen et al.'s recommendation for harmonizing injection-acquisition time delay, scanning direction, and whole-body scan duration, to reliably compare TBRs between centers. Furthermore, the authors acknowledged that TBR repeatability should be investigated for treatment-response assessment. We suggest that TBR repeatability should take into account repeatability of both tumor- and blood-activity concentration because $\operatorname{TBR}(\mathrm{t})=\mathrm{C}_{\mathrm{T}}(\mathrm{t}) / \mathrm{C}_{\mathrm{p}}(\mathrm{t})\left(=\mathrm{SUV}_{\text {tumor }} / \mathrm{SUV}_{\text {blood }}\right)$, as demonstrated with SUV in lung cancer patients investigated with ${ }^{18} \mathrm{~F}-\mathrm{FDG}$ (3). Despite this increased repeatability percentage, blood normalization involved in the TBR makes it a much better surrogate for Ki than the SUV, because the latter is significantly affected by the difference in the blood-activity concentration between patients, depending on the total tumor burden, as illustrated in Figure 4 in Jansen et al. $(1,4)$. This SUV feature involving TBR may be simply summarized as: $\mathrm{SUV}_{\text {tumor }}(\mathrm{t})=\operatorname{TBR}(\mathrm{t}) \times \mathrm{SUV}_{\text {blood }}(\mathrm{t}) \propto \operatorname{TBR}(\mathrm{t}) \times \mathrm{C}_{\mathrm{p}}(\mathrm{t})$. Finally,

COPYRIGHT @ 2019 by the Society of Nuclear Medicine and Molecular Imaging. let us note that, because of decay correction, the SUV apparently rises during the first $2 \mathrm{~h}$ after injection (assuming irreversible trapping), whereas the TBR actually rises with time (Fig. 3A), furthermore justifying Jansen et al.'s recommendation (1).

In conclusion, we are convinced that the image-based TBR can reliably assess ${ }^{18} \mathrm{~F}$-DCFPyL uptake in prostate cancer metastases, thus opening up possible tumor characterization and treatmentresponse assessment. We suggest that the TBR may be considered a simplified Patlak's analysis that is adapted to daily clinical practice (i.e., to standard whole-body acquisition without the need for invasive blood sampling). In this connection, we suggest that reporting the correlation between TBR and Patlak's Ki might be of interest.

\section{REFERENCES}

1. Jansen BHE, Yaqub M, Voortman J, et al. Simplified methods for quantification of ${ }^{18}$ F-DCFPyL uptake in patients with prostate cancer. J Nucl Med. April 18, 2019 [Epub ahead of print].

2. Patlak CS, Blasberg RG, Fenstermacher JD. Graphical evaluation of blood-to brain transfer constants from multiple-time uptake data. J Cereb Blood Flow Metab. 1983;3:1-7.

3. Laffon E, Milpied N, Marthan R. Measurement uncertainty of lesion and reference mediastinum standardized uptake value in lung cancer. Nucl Med Commun. 2017;38:509-514.

4. Laffon E, Marthan R. The total amount of uptake may affect the input function: a theoretic approach about ${ }^{18}$ F-FDG PET imaging. Nucl Med Biol. 2015;42:724-727.

Eric Laffon*
Henri de Clermont
Roger Marthan

Published online Aug. 26, 2019.

DOI: 10.2967/jnumed.119.232926

\section{Reply: Quantification of ${ }^{18}$ F-DCFPyL Uptake: TBR Versus Patlak's Analysis}

REPLY: We thank Drs. Laffon, de Clermont, and Marthan for their positive letter on our paper $(1,2)$. They effectively argue that the tumor-to-blood ratio (TBR) can be seen as a simplification of a Patlak's analysis - which in turn should be seen as a simplification of full pharmacokinetic analysis (based on nonlinear regression analysis with arterial blood sampling as input function). As suggested by Laffon et al., we have provided the correlation between Patlak's $\mathrm{K}_{\mathrm{i}}$ (net-influx-rate constant) and our proposed image-based TBR (Fig. 1) (2). Indeed, a good correlation was observed $\left(R^{2}=0.91\right)$, supporting the suggestion of Laffon et al. that TBR could be considered as a surrogate for Patlak's analysis that is suitable for daily clinical practice.

Overall, our study and the letter by Laffon et al. $(1,2)$ emphasize the need for technical validation of simplified metrics to quantify tracer uptake of novel tracers. In particular, our study demonstrated that SUV, normalized to injected activity over body weight, is not a 


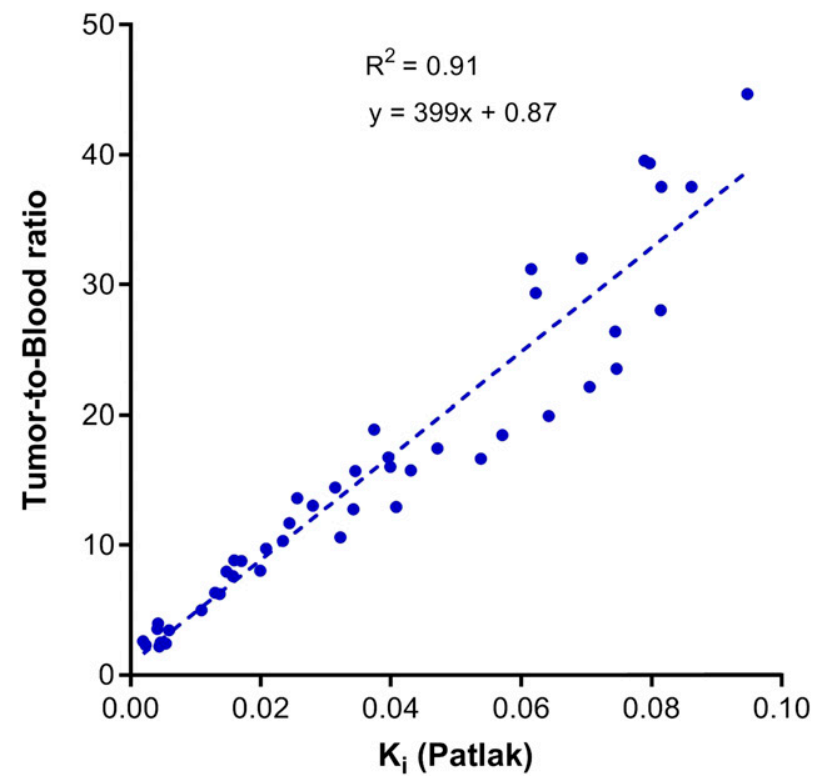

FIGURE 1. Correlation of image-based tumor-to-blood ratios and $\mathrm{K}_{\mathrm{i}}$ derived from Patlak's graphical analysis.

robust and valid simplified method for ${ }^{18} \mathrm{~F}-\mathrm{DCFPyL}$ uptake quantification-despite its widespread (often unvalidated) use. The main reason for the invalidity of SUV is that the input function, that is, the bioavailability of the tracer in plasma to tissue, is not comparable between subjects. Normalizing tracer uptake by injected activity over body weight (or lean body mass) assumes that the input function of individual patients is simply a scaled version of a population curve. When this assumption is violated, quantitative kinetic approaches that include an individually measured input function, such as Patlak analysis, are required. A simplification to the Patlak approach could be to normalize the tumor uptake to blood activity concentrations, as was shown in our paper. The use of TBR, at least partly, compensates for changes in the input functions that are not explained by variation in injected activity and weight alone. In our specific case, the overall mass of the disease affected the shape and amplitude of the input function, and thus normalizing tumor uptake by injected activity over weight, that is, SUV, should not be used for the quantification of ${ }^{18} \mathrm{~F}$-DCFPyL uptake.

Lastly, we agree with Laffon et al. that not only the repeatability of tumor uptake (SUV) should be evaluated, but also the repeatability of the TBR (and the blood-activity concentration itself) must be understood. The same differences in tracer bioavailability that were observed between patients can develop within patients over time, in the case of disease progression or treatment response. Results on our repeatability study are expected shortly.

\section{REFERENCES}

1. Laffon E, de Clermont H, Marthan R. Quantification of ${ }^{18} \mathrm{~F}$-DCFPyL uptake: TBR versus Patlak's analysis [Letter]. J Nucl Med. 2019;60:1834.

2. Jansen BHE, Yaqub M, Voortman J, et al. Simplified methods for quantification of ${ }^{18}$ F-DCFPyL uptake in patients with prostate cancer. J Nucl Med. April 18, 2019 [Epub ahead of print].

Bernard H.E. Jansen Maqsood Yaqub Matthijs C.F. Cysouw André N. Vis

Reindert J.A. van Moorselaar N. Harry Hendrikse Otto S. Hoekstra
Ronald Boellaard

Daniela E. Oprea-Lager*

*Amsterdam University Medical Center (VU University)

De Boelelaan 1117, Room 1F-012

Amsterdam, $1081 \mathrm{HV}$, Netherlands

E-mail:d.oprea-lager@vumc.nl

Published online Aug. 26, 2019.

DOI: 10.2967/jnumed.119.234047

\section{Off-Target Report on ${ }^{18}$ F-Sodium Fluoride PET/CT for Detection of Skeletal Metastases in Prostate Cancer}

TO THE EDITOR: In a recent report in the Journal of Nuclear Medicine, cited by AuntMinnie, Zacho et al. found, according to the title of their communication, "No added value of ${ }^{18} \mathrm{~F}$-sodium fluoride $\left({ }^{18} \mathrm{~F}-\mathrm{NaF}\right) \mathrm{PET} / \mathrm{CT}$ for the detection of bone metastases in patients with newly diagnosed prostate cancer with normal bone scintigraphy" (1). In 81 intermediate- or high-risk prostate cancer patients with negative bone scintigraphy scheduled for prostatectomy, ${ }^{18} \mathrm{~F}-\mathrm{NaF}$ PET/CT "indicated bone metastasis" in 1 and was equivocal in 7 patients. None of these patients exhibited biochemical failure (prostate-specific antigen level $\geq 2 \mathrm{ng} / \mathrm{mL} 6 \mathrm{wk} / 6 \mathrm{mo}$ after radical prostatectomy), whereas all 6 patients with biochemical failure had negative ${ }^{18} \mathrm{~F}-\mathrm{NaF}$ PET/CT (and negative bone scintigraphy) findings making the authors conclude as stated in their title.

Their report is off-target because (1) skeletal metastases are bone marrow and not bone metastases and (2) neither ${ }^{18} \mathrm{~F}-\mathrm{NaF}$ PET/CT nor bone scintigraphy mirror bone marrow metastases, but late-occurring bone changes that may or may not be due to active cancerous processes $(2,3)$. As in other recent communications $(4,5)$, the authors disregarded the true nature of skeletal metastases, which home and grow in the bone marrow long before they give rise to structural changes in the osseous bone substance that can be detected by bone scintigraphy, ${ }^{18} \mathrm{~F}-\mathrm{NaF}$ PET/CT, or other imaging modalities. This was highlighted more than $10 \mathrm{y}$ ago by Basu et al. $(6,7)$ and has recently given rise to comments in both the Journal of Nuclear Medicine and the European Journal of Nuclear Medicine and Molecular Imaging (2,3), the latter calling for a much needed paradigm shift, since we cannot go on using methods unable to fulfil their stated purpose and that, therefore unfortunately, may lead to inappropriate patient management.

The reason why Zacho et al. did not observe an association between biochemical failure and abnormal ${ }^{18} \mathrm{~F}-\mathrm{NaF}$ PET/CT findings is a simple one: there should not be an association-at least not a very close one. An increase in prostate-specific antigen, however unspecific, is usually a reaction to cancer cells that are still present and growing after prostatectomy. However, this may have little to do with what is seen by ${ }^{18} \mathrm{~F}-\mathrm{NaF}$ PET/CT or bone scintigraphy, since both methods depict unspecific structural changes in osseous tissue that occur late in the development of skeletal metastasis and remain unchanged for a long time after the cancer may have disappeared, for instance, due to effective chemo- or radiation therapy $(2,3)$. Thus, it is time to realize that all imaging modalities demonstrating structural bone changes are not reliable harbingers of skeletal metastases and should be abandoned in favor of ${ }^{18} \mathrm{~F}$-FDG PET/CT and, when it comes to prostate cancer, perhaps PSMA PET/CT. Time will show which of the latter 2 approaches are preferable for showing bone marrow metastases in prostate cancer. 